\title{
Special issue on applications of variational inequality problems
}

\author{
Mohamed A. Tawhid
}

Published online: 28 June 2012

(C) Springer Science+Business Media, LLC 2012

I am very pleased to see the completion of this special issue of Optimization and Engineering: Applications of Variational Inequality Problems. Over the past several decades, variational inequalities have attracted a great deal of attention from both researchers and practitioners.

It is well known that variational inequalities cover diverse disciplines in partial differential equations, optimal control, optimization, mathematical programming, mechanics, finance, operations research, engineering (dynamic rigid- body model, nonlinear obstacle problems), economics equilibrium models, and science.

This special issue focus on applications of variational inequality problems. It includes 10 papers selected after a peer revision. The summaries of the 10 papers in this issue are listed below:

The first paper, by Laura Scrimali, discusses time-dependent spatial price equilibrium problem. The author presents two different perspectives. First a useroptimization time-dependent model is studied. In particular, the equilibrium conditions are stated and the equivalence between the problem under consideration and an evolutionary variational inequality is proved. Afterwards, introducing control policies, the problem becomes a time-dependent spatial price equilibrium control problem from the policy-maker's point of view and it is formulated as an evolutionary

M.A. Tawhid (凶)

Thompson Rivers University, Kamloops, BC, Canada

e-mail:mtawhid@tru.ca

M.A. Tawhid

Alexandria University, Alexandria, Egypt

M.A. Tawhid

Egypt-Japan University of Science \& Technology, Alexandria, Egypt 
inverse variational inequality. Moreover, the author gives a classical variational inequality formulation of the optimal control problem in order to use the results in literature in both theory. Finally, some existence results and a numerical example are provided.

The second paper, by Gonzalo Alduncin, investigates the well-posedness of an evolutionary problem and stationary problem arising from control nonlinear diffusion macro-hybrid mixed variational problems by using some abstract theory of evolution equations and convex analysis. The results seem to attract interest of researchers working on nonlinear diffusions and abstract theories of evolution equations.

The third paper, by E. Allevi, A. Gnudi, and I.V. Konnov, presents an equilibrium model for multi-period spatial auction markets. In particular, the auction markets are modeled using a monotone function in the variational inequality model. Computational methods for strictly monotone and non-strictly monotone cost functions are discussed, and numerical experiments are presented.

The fourth paper, by P. Falsaperla, F. Raciti, and L. Scrimali, studies the multicriteria spatial price network equilibrium model in which consumers are allowed to weight both the transportation cost and the transportation time associated with the shipment of a given commodity. In particular, the authors assume that weights are not deterministic but subject to random fluctuations. Also, they formulate the problem as a random variational inequality and prove that under natural assumptions the associated random operator satisfies a uniform monotonicity property.

The fifth paper, N. Krishnamurthy, T. Parthasarathy, and G. Ravindran give a survey of some results on the orderfield property of 2-player and multi-player stochastic games and their mixtures. Some of these classes of stochastic games can be solved by formulating them as a Linear Complementarity Problem (LCP) or (Generalized) Vertical Linear Complementarity Problem (VLCP). The authors discuss some of these results and prove that certain new subclasses and mixtures of multi-player (or nperson) stochastic games can be solved via LCP formulations.

The sixth paper, by Zongxia Liang, develops variational inequality approach to establish optimal stopping problems arising from the pricing of stock loan and capped stock loan models. The valuations of the stock loans and ranges of reasonable values of key parameters such as loan sizes, interest rates, fees for providing such a service and quantity of this automatic termination clause and relationships among these parameters as well as the optimal terminable stopping times are explicitly derived. The paper also gives two numerical samples to portray the results and the difference between stock loan model and capped stock loan model.

The seventh paper, by Zugang Liu and Anna Nagurney, presents a multitiered dynamic supply chain network equilibrium modeling framework in which the decisionmakers have sufficient information about the future and seek to determine their optimal plans that maximize their profits over the multiperiod planning horizon. The authors construct the finite-dimensional variational inequality governing the equilibrium of the multiperiod competitive supply chain network. Also, the authors investigate the interplay of the heterogeneous decision-makers in the supply chain in a dynamic setting, and compute the resultant equilibrium pattern of product outputs, transactions, inventories, and product prices. Moreover, they establish the supernetwork equivalence of the multiperiod supply chain model with a properly configured 
transportation network, which provides a new interpretation of the equilibrium conditions of the former in terms of paths and path flows. Numerical examples are provided to illustrate how such multiperiod supply chain problems can be reformulated and solved as transportation network equilibrium problems in practice.

The eighth paper, Monica G. Cojocaru investigates the evolution of a class of noncooperative games. The main idea is to unify the evolutionary variational inequalities with a hybrid dynamical system framework. The paper begins with an introduction of vaccinating games and evolutionary variational inequalities. The author formulates the dynamic games in the terms of game theory and gives a detailed discussion of hybrid systems. The efficiency of the hybrid system approach is used to study the evolution of population groups' strategies engaged in a noncooperative vaccinating game over a finite timer interval.

The ninth paper, by Annamaria Barbagallo, studies a general evolutionary traffic network where the author introduces some delay effects. In particular, starting from the weighted quasi-variational in-equalities, which are useful to characterize the congested traffic equilibrium problem, the author addresses the retarded weighted network proving the equivalence between the equilibrium conditions and the generalized Wardrop's principle. The main result of the paper is that the increasing of the weight on a path causes the decreasing of flow on that path and, analogously, the increasing of the weight on the path cost function causes an increasing on the path cost. Hence the introduction of weights leads to reduce the flow on the more expensive paths.

The last paper, by Giovanni Anello, Maria B. Donato, and Monica Milasi, studies an existence result of competitive equilibrium points in a Walrasian pure exchange economy involving quasi-concave utility functions. The competitive equilibrium conditions are equivalent to a generalized quasi-variational inequality, then by using a variational approach, the authors are able to achieve the existence of competitive equilibrium points. The aim of this paper is to weaken the concavity condition on utility functions by assuming that they are quasi-concave. This paper is published in advanced in Optim. Eng. 13(2), pp. 169-179 (2012).

As a final note, I would like to express my gratitude to all the authors for their contribution and collaboration, and to the many reviewers, who generously participated in the elaboration of this issue, for their comments, suggestions and timely responses. I would also like to extend my sincere thanks to the Editor-in-Chief, Prof. Tamas Terlaky, for his support for this focused issue. Special thanks are also due to Journal Editorial Office, for providing valuable technical and administrative support, during the reviewing process.

Guest editor

Mohamed A. Tawhid 